\title{
Localized Digestive System Neuroendocrine Tumor G1
}

National Cancer Institute

\section{Source}

National Cancer Institute. Localized Digestive System Neuroendocrine Tumor G1. NCI Thesaurus. Code C7861.

A well differentiated neuroendocrine tumor (carcinoid tumor) that arises from the digestive system and is restricted to the site of origin without evidence of metastasis. 